\title{
Modeling of nonlinear variable viscosity on peristaltic transport of fluid with slip boundary conditions: Application to bile flow in duct
}

\author{
S. Kumari*, T. K. Rawat, S. P. Singh \\ Department of Mathematics, Dayalbagh Educational Institute, Dayalbagh, Agra- 282005, Uttar \\ Pradesh, India
}

Received 17 March 2021, accepted in final revised form 10 June 2021

\begin{abstract}
The present article deals with variable viscosity on the peristaltic transport of bile in an inclined duct under the action of slip boundary conditions. The wall geometry is described by the sinusoidal wave propagating in the axial direction with different amplitude and with constant speed. The flow of fluid is examined in a wave frame of reference, moving with the velocity of the wave. Mathematical modeling of the problem includes equations of motion and continuity. The fluid flow is investigated by converting the equations into a nondimensionalized form simplified considering long wavelength and low Reynolds number approximation. The analytic expressions for axial velocity, pressure gradient, and pressure rise over a single wavelength cycle are obtained. The impact of various parameters such as slip parameter, viscosity parameter, angle of inclination, gravity parameter and amplitude ratio on axial velocity, pressure gradient and pressure rise are discussed in detail by plotting graphs in MATLAB R2018b software. In this article, a comparison of linear and nonlinear variation of viscosity of bile has been made. It is concluded that velocity and pressure rise is more in case linear variation of viscosity, whereas more pressure gradient is required in case of nonlinear variation of viscosity.
\end{abstract}

Keywords: Peristaltic motion; Variable viscosity; Knudsen number; Inclined channel.

(c) 2021 JSR Publications. ISSN: 2070-0237 (Print); 2070-0245 (Online). All rights reserved. doi: http://dx.doi.org/10.3329/jsr.v13i3.52487 J. Sci. Res. 13 (3), 821-832 (2021)

\section{Introduction}

Bile is one of the bio-fluids in the human body. Peristaltic plays an important role in bile transportation from the gallbladder to the first part of the duodenum, i.e., the small intestine. The peristaltic word refers to the wavy (i.e. the contraction and expansion) of the walls and a tube-like structure that contains fluid. Peristaltic is an important factor of fluid for transporting it in many biological systems such as smooth muscle tubes, the bile duct, the ureter, swallowing of food through the esophagus, stomach and intestine. Peristaltic waves can be small, long, continuous depending upon the wall of the organ. Nowadays, many artificial mechanical devices are developed on the basis of the principle of peristaltic pumping for transporting fluid, for example, blood pump machine, dialysis

\footnotetext{
*Corresponding author: shivangis898@gmail.com
} 
machine, heart-lung machine, etc. Previously many authors have studied peristaltic motion using theoretical, experimental and numerical approaches. Many theoretical studies have been done on peristaltic transport of various physiological fluids to analyze the behavior of fluid in different conditions using analytical, computational and experimental techniques. The analysis was restricted to examine small peristaltic wave amplitude and the assumption that fluid is free. The first research on the peristaltic transport of viscous fluid was presented by Latham [1]. Thereafter, many authors devoted their studies toward the peristaltic motion of the fluid. The influence of viscosity variation on the peristaltic motion of a Newtonian fluid through an asymmetric tube is studied by Hayat and Ali [2] and the study reveals that wave amplitude is also affected by peristaltic flow with the mean flow parameter. Muthu et al. [3] presented a mathematical model for the peristaltic motion of micropolar fluid in circular cylindrical elastic wall tubes under the effect of wall properties; the model is solved using the perturbation method for small values of amplitude ratio. Elmaboud et al. [4] theoretically examined the peristaltic transport of incompressible Newtonian fluid with variable viscosity in a finite length tube; perturbation technique is taken out to obtain results. Akbar et al. [5] analyzed the impact of heat transfer with partial slip-on Williamson fluid in an inclined channel. Kavitha et al. [6] proposed a model for peristaltic flow of Jeffrey fluid in contact with Newtonian fluid through an inclined asymmetric channel under the assumption of long wavelength and low Reynolds number approximation. Tanveer et al. [7] explored the study of variable viscosity of peristaltic flow of Sisko fluid in a curved channel with wall slip condition. Khan et al. [8] analyzed the peristaltic transport of an incompressible non-Newtonian fluid through a porous medium in an inclined finite length tube with slip boundary conditions.

Bile is one of the biofluids produced continuously by the liver responsible for emulsifying fat (lipids) in the duodenum (i.e., the small intestine). The daily secreted amount of bile in the human biliary system is approximately one liter. The biliary system consists of the liver gallbladder, biliary ducts (common bile duct, hepatic duct, and cystic duct) and the structure of sphincters. Biliary ducts are the medium of transportation of bile in the biliary system. The gallbladder is a pear-shaped organ located directly below the liver and regulates the bile flow. Bile plays an essential role in absorbing protein, vitamin $\mathrm{D}, \mathrm{E}, \mathrm{K}$ and $\mathrm{A}$ that are soluble in lipids.

There are only a few papers devoted to the rheological properties of bile flow modeling in different conditions of the biliary system using the analytical, computational and experimental method without considering the peristaltic motion. Gottschalk and Lochner [9] examined 33 samples and reported that post-operative T-tube bile is Maxwell fluid. Atabi et al. [10] investigated the flow of bile in the patient-specific cystic duct using an experimental approach and revealed that the presence of gallstones might lead to increase resistance. They also compared clinical and CFD (Computational Fluid Dynamics) resulted of bile in the human cystic duct and showed CFD is a relevant mechanism to investigate the function of the biliary system [11]. Kuchumov et al. [12] presented an experimental investigation and CFD simulation of the non-Newtonian flow 
of pathological bile in the biliary system and it was observed that at high shear stress, pathological bile behaves like a Newtonian fluid. Luo et al. [13] worked to understand the biomechanical behavior of the human biliary system. Ooi et al. [14] studied bile flow in the human cystic duct and found a great influence of valve of heister on the resistance to bile flow. Kuchumov et al. [15] presented a peristaltic flow of bile as Carreau's fluid in the stenosed duct. They also presented a model for pathological bile flow in the major duodenal papilla duct with stone and it was shown that pathological bile behaves like a non-Newtonian fluid [16]. Kuchumov [17] developed a model for peristaltic lithogenic bile flow through a finite length tapered duct in the presence of papillary stenosis. Maiti and Misra [18] put forward a mathematical model for the peristaltic motion of a fluid in a porous channel as an application to bile flow in a pathological state and concluded that in the presence of gallstone, bile velocity increases as the porosity parameter increases. Gujral and Singh [19] analyzed the flow parameters of blood in the presence of overlapping stenosis while considering the axial variation of viscosity using a power-law non-Newtonian model. They also compared linear and quadratic variations of viscosity. Peristaltic transport of Ellis fluid in a vertical channel with slip conditions is studied by Goud et al. [20]. Mahmood et al. [21] worked on the peristaltic motion of a couple of stress fluids while considering lubrication effects.

The purpose of this article is to present a mathematical model that can be considered as an application of bile flow in a duct. Peristaltic transport of Newtonian fluid in an inclined duct under the impact of variable viscosity with slip boundary conditions has been investigated. The governing equations of motion and continuity are solved using relevant boundary conditions and simplified by long wavelength and low Reynolds number approximation. Analytical expressions of axial velocity, pressure gradient and pressure rise are obtained for small values of viscosity parameter and analyzed by plotting in MATLAB R2018b software. The article is organized as follows. Section 2 and section 3 contain mathematical formulation and solution of the problem. Section 4 relates results and discussion and concluding remarks are presented in section 5. The aim of this article is to study the role of fluid dynamics in the human biliary system. From this article, it is expected that obtained results represented here will serve as a good theoretical model which can be used to understand the flow mechanism of peristaltic transport of bile in the human biliary system for a real-world problem.

\begin{tabular}{|cl|}
\hline Nomenclature & \\
$(\bar{X}, \bar{Y})$ & Cartesian co-ordinate in fixed wave frame \\
$(\bar{U}, \bar{V})$ & velocity components along with $\bar{X}$ and $\bar{Y}$ directions \\
$\left(x^{*}, y^{*}\right)$ & cartesian co-ordinate in a wave frame of reference \\
$\left(u^{*}, v^{*}\right)$ & velocity components along $x^{*}$ and $y^{*}$ directions \\
$c$ & wave speed \\
$p^{*}$ & fluid pressure \\
$g$ & acceleration due to gravity \\
$R e$ & Reynolds number \\
\hline
\end{tabular}




\begin{tabular}{|ll|}
\hline$K_{n}$ & velocity slip parameter \\
$\alpha$ & Reynolds model viscosity parameter \\
$\gamma$ & angle of inclination \\
$\delta$ & wave number \\
$\eta$ & gravity parameter \\
$\lambda$ & wavelength \\
$\bar{\mu}$ & variable viscosity \\
$\mu_{0}$ & constant viscosity \\
$\rho$ & density of fluid \\
\hline
\end{tabular}

\section{Mathematical Formulation}

Let us consider the peristaltic transport of incompressible Newtonian fluid (Bile) with variable viscosity and slip property in the two-dimensional inclined symmetric channel (duct). The wall geometry is described as the wavy motion of a fluid (Bile) along the wall. The wavelength is comparable with the channel (duct) length; thus, the wavenumber is small and the Reynolds number is negligible.

The wall geometry is defined as follow [6]

$$
\bar{H}(\bar{X}, \bar{t})=a+b \sin \frac{2 \pi}{\lambda}(\bar{X}-c \bar{t})
$$

where $b$ is the amplitude of the peristaltic wave, $\lambda$ is the wavelength, $c$ is wave velocity, $a$ is an inlet radius, $\bar{t}$ is time and $(\bar{X}, \bar{Y})$ is the cartesian co-ordinate in a fixed wave frame.

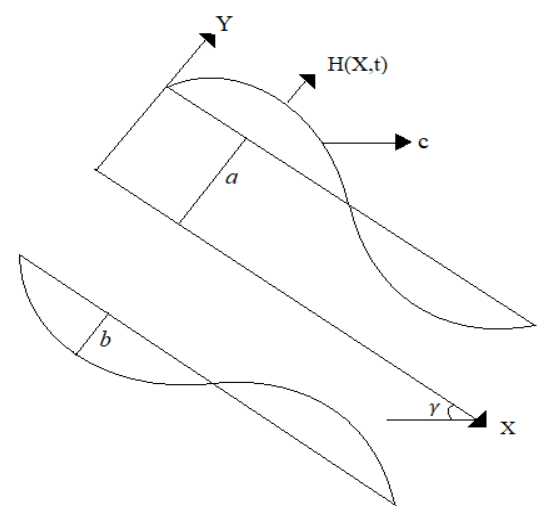

Fig. 1. Physical sketch of the inclined duct.

The governing equations of motion are [22]

$$
\begin{aligned}
& \frac{\partial \bar{U}}{\partial \bar{X}}+\frac{\partial \bar{V}}{\partial \bar{Y}}=0 \\
& \rho\left(\frac{\partial \bar{U}}{\partial \bar{t}}+\bar{U} \frac{\partial \bar{U}}{\partial \bar{X}}+\bar{V} \frac{\partial \bar{U}}{\partial \bar{Y}}\right)=-\frac{\partial \bar{P}}{\partial \bar{X}}+2 \frac{\partial}{\partial \bar{X}}\left(\bar{\mu}(\bar{Y}) \frac{\partial \bar{U}}{\partial \bar{X}}\right)+\frac{\partial}{\partial \bar{Y}}\left(\bar{\mu}(\bar{Y})\left(\frac{\partial \bar{V}}{\partial \bar{X}}+\frac{\partial \bar{U}}{\partial \bar{Y}}\right)\right)+\rho g \sin \gamma
\end{aligned}
$$




$$
\rho\left(\frac{\partial \bar{U}}{\partial \bar{t}}+\bar{U} \frac{\partial \bar{V}}{\partial \bar{X}}+\bar{V} \frac{\partial \bar{V}}{\partial \bar{Y}}\right)=-\frac{\partial \bar{P}}{\partial \bar{Y}}+2 \frac{\partial}{\partial \bar{Y}}\left(\bar{\mu}(\bar{Y}) \frac{\partial \bar{V}}{\partial \bar{Y}}\right)+\frac{\partial}{\partial \bar{X}}\left(\bar{\mu}(\bar{Y})\left(\frac{\partial \bar{V}}{\partial \bar{X}}+\frac{\partial \bar{U}}{\partial \bar{Y}}\right)\right)-\rho g \cos \gamma
$$

Here we introduce a wave frame of reference $\left(x^{*}, y^{*}\right)$ moving with the velocity $c$, where the motion becomes independent of time $(t)$.

The transformation from the fixed wave frame $(\bar{X}, \bar{Y})$ to the wave frame of reference $\left(x^{*}, y^{*}\right)$ is given by

$$
x^{*}=\bar{X}-c t, y^{*}=\bar{Y}, u^{*}=\bar{U}-c, v^{*}=\bar{V}, p^{*}=\bar{P}
$$

Where $u^{*}, v^{*}$ and $\bar{U}, \bar{V}$ are the velocity components in the fixed wave frame and wave frame of reference, respectively.

Now let us consider the following non-dimensional variables.

$$
\begin{aligned}
& x=\frac{x^{*}}{\lambda}, y=\frac{y^{*}}{a}, u=\frac{u^{*}}{c}, v=\frac{\lambda v^{*}}{a c}, p=\frac{a^{2} p^{*}\left(x^{*}\right)}{\lambda \mu c}, t=\frac{c t^{*}}{\lambda}, h(x)=\frac{\bar{H}\left(x^{*}\right)}{a}, \mu(y)=\frac{\mu^{*}\left(y^{*}\right)}{\mu_{0}}, \\
& q=\frac{q^{*}}{a c}
\end{aligned}
$$

Where $\mu_{0}$ is the constant viscosity and $\delta$ is the wavenumber.

Using equation (6), equations (2) - (4) converted into

$$
\begin{gathered}
\frac{\partial u}{\partial x}+\frac{\partial v}{\partial y}=0 \\
\operatorname{Re} \delta\left((u+1) \frac{\partial u}{\partial x}+\frac{\partial u}{\partial y}\right)=-\frac{\partial p}{\partial x}+2 \delta^{2} \frac{\partial}{\partial x}\left(\mu(y) \frac{\partial u}{\partial x}\right)+\frac{\partial}{\partial y}\left(\mu(y)\left(\delta^{2} \frac{\partial v}{\partial x}+\frac{\partial u}{\partial y}\right)\right)+\eta \sin \gamma \\
\operatorname{Re}^{3}\left((u+1) \frac{\partial v}{\partial x}+\frac{\partial v}{\partial y}\right)=-\frac{\partial p}{\partial y}+2 \delta^{2} \frac{\partial}{\partial x}\left(\mu(y) \frac{\partial v}{\partial y}\right)+\delta^{2} \frac{\partial}{\partial x}\left(\mu(y)\left(\delta^{2} \frac{\partial v}{\partial x}+\frac{\partial u}{\partial y}\right)\right)-\eta \delta \cos \gamma
\end{gathered}
$$

Where $\delta$ and $R e$ are the non-dimensional parameters, called wave number and Reynolds number respectively and are given by

$$
\delta=\frac{a}{\lambda}, R e=\frac{\rho a c}{\mu_{0}}
$$

Considering long wavelength $(\delta \ll 1)$ and low Reynolds number $(R e \rightarrow 0)$, equations (8) and (9) becomes

$$
\begin{aligned}
& \frac{\partial p}{\partial x}-\eta \sin \gamma=\frac{\partial}{\partial y}\left[\mu(y) \frac{\partial u}{\partial y}\right] \\
& \frac{\partial p}{\partial y}=0
\end{aligned}
$$

It is observed from equation (12), $p$ is not a function of $y$, therefore equation (11) takes the form

$$
\frac{d p}{d x}-\eta \sin \gamma=\frac{\partial}{\partial y}\left[\mu(y) \frac{\partial u}{\partial y}\right]
$$

For the present article, the dimensionless variation of viscosity is taken in the form of $\mu(y)=e^{-\alpha y}$ where $(\alpha \ll 1)$. where $\alpha$ is the Reynolds model viscosity parameter [22].

$$
\frac{d p}{d x}-\eta \sin \gamma=\frac{\partial}{\partial y}\left[e^{-\alpha y} \frac{\partial u}{\partial y}\right]
$$

Dimensionless boundary conditions for the velocity are 


$$
\begin{aligned}
& \frac{\partial u}{\partial y}=0 \quad \text { at } \quad y=0 \\
& u=-K_{n} \frac{\partial u}{\partial y} \quad \text { at } y=h
\end{aligned}
$$

Where $K_{n}$ is velocity slip parameter, called Knudsen number.

The dimensionless volume flow rate in wave frame of reference is given by

$$
q=\int_{0}^{h} u d y
$$

The dimensionless instantaneous flux in the fixed wave frame is

$$
Q(x, t)=\int_{0}^{h}(u+1) d y=q+h
$$

The time-average flux $\Theta$ over one wave period of peristaltic wave is given by

$$
\Theta=\frac{1}{T} \int_{0}^{T} Q d t=\int_{0}^{1}(q+h) d x=q+1
$$

\section{Solution of the Problem}

In direction to understand the effect of variable viscosity on peristaltic transport of bile, two cases are taken into account

\section{Case 1: Linear variation of viscosity}

The dimensionless variation of viscosity is taken in the form of $\mu(y)=1-\alpha y$ where $(\alpha \ll 1)$.

$$
\frac{d p}{d x}-\eta \sin \gamma=\frac{\partial}{\partial y}\left[(1-\alpha y) \frac{\partial u}{\partial y}\right]
$$

Solving equation (20) with the respective boundary conditions (15) and (16), we obtain

$$
u=\left(\frac{d p}{d x}-\eta \sin \gamma\right)\left[\frac{h}{\alpha}+\frac{\log |1-\alpha h|}{\alpha^{2}}-\frac{y}{\alpha}-\frac{\log |1-\alpha y|}{\alpha^{2}}\right]
$$

and the volume flow rate $q$ is given by

$$
q=\left(\frac{d p}{d x}-\eta \sin \gamma\right)\left[\frac{h}{\alpha^{2}}+\frac{h^{2}}{2 \alpha}-\frac{2 h \log |1-\alpha h|}{\alpha^{2}}+\frac{\log |1-\alpha h|}{\alpha^{3}}\right]
$$

From equation (22), we obtain

$$
\frac{d p}{d x}=\frac{q}{\left[\frac{h}{\alpha^{2}}+\frac{h^{2}}{2 \alpha}-\frac{2 h \log |1-\alpha h|}{\alpha^{2}}+\frac{\log |1-\alpha h|}{\alpha^{3}}\right]}+\eta \sin \gamma
$$

Pressure rise per wavelength can be written by

$$
\Delta P=\int_{0}^{1} \frac{d p}{d x} d x=\int_{0}^{1}\left(\frac{q}{\left[\frac{h}{\alpha^{2}}+\frac{h^{2}}{2 \alpha}-\frac{2 h \log |1-\alpha h|}{\alpha^{2}}+\frac{\log |1-\alpha h|}{\alpha^{3}}\right]}+\eta \sin \gamma\right) d x
$$

\section{Case 2: Nonlinear variation of viscosity}

The dimensionless variation of viscosity is taken in the form of $\mu(y)=1-\alpha y+\frac{\alpha^{2} y^{2}}{2 !}$ where $(\alpha \ll 1)$. 


$$
\frac{d p}{d x}-\eta \sin \gamma=\frac{\partial}{\partial y}\left[\left(1-\alpha y+\frac{\alpha^{2} y^{2}}{2 !}\right) \frac{\partial u}{\partial y}\right]
$$

Solving equation (25) with the respective boundary conditions (15) and (16), we obtain

$$
\begin{aligned}
& u=\left(\frac{d p}{d x}-\eta \sin \gamma\right) \frac{1}{\alpha^{2}}\left[\log \left|1-\alpha y+\frac{\alpha^{2} y^{2}}{2 !}\right|+2 \tan ^{-1}(\alpha y-1)-\log \mid 1-\alpha h+\right. \\
& \left.\frac{\alpha^{2} h^{2}}{2 !} \mid-2 \tan ^{-1}(\alpha h-1)\right]
\end{aligned}
$$

and the volume flow rate $q$ is given by

$$
\begin{aligned}
& q=\frac{1}{\alpha^{2}}\left(\frac{d p}{d x}-\eta \sin \gamma\right) \int_{0}^{h}\left[\log \left|1-\alpha y+\frac{\alpha^{2} y^{2}}{2 !}\right|+2 \tan ^{-1}(\alpha y-1)-\log \mid 1-\alpha h+\right. \\
& \left.\frac{\alpha^{2} h^{2}}{2 !} \mid-2 \tan ^{-1}(\alpha h-1)\right] d y
\end{aligned}
$$

From equation (27), we obtain

$$
\frac{d p}{d x}=\frac{q \alpha^{2}}{\int_{0}^{h}\left[\log \left|1-\alpha y+\frac{\alpha^{2} y^{2}}{2 !}\right|+2 \tan ^{-1}(\alpha y-1)-\log \left|1-\alpha h+\frac{\alpha^{2} h^{2}}{2 !}\right|-2 \tan ^{-1}(\alpha h-1)\right] d y}+\eta \sin \gamma
$$

Pressure rise per wavelength can be written by

$$
\begin{aligned}
& \Delta P=\int_{0}^{1} \frac{d p}{d x} d x=\int_{0}^{1}\left[\frac{q}{\int_{0}^{h} \frac{1}{\alpha^{2}}\left[\log \left|1-\alpha y+\frac{\alpha^{2} y^{2}}{2 !}\right|+2 \tan ^{-1}(\alpha y-1)-\log \left|1-\alpha h+\frac{\alpha^{2} h^{2}}{2 !}\right|-2 \tan ^{-1}(\alpha h-1)\right] d y}+\right. \\
& \eta \sin \gamma] d x
\end{aligned}
$$

\section{Results and Discussion}

This section is devoted to analyze the influence of various parameters, namely velocity slip parameter $K_{n}$, viscosity parameter $\alpha$, angle of inclination $\gamma$, gravity parameter $\eta$ and amplitude ratio $\phi$ on the derived distributions axial velocity $u$, pressure gradient $\frac{d p}{d x}$ and pressure rise $\Delta P$ for fluid (bile). Velocity profiles are displayed from graphs 2(a)-2(d) for different values of effecting parameters of the problem velocity slip parameter $K_{n}$, viscosity parameter $\alpha$, angle of inclination $\gamma$ and gravity parameter $\eta$, respectively. These Figs. represent the relation between axial velocity $u$ and the axial distance $x$. It is also found in all plotted graphs that bile velocity is more in the case of linear variation of viscosity than the nonlinear variation of viscosity. Fig. 2(a) is constructed to see the deviation of axial velocity for distinct values of $K_{n}$. Velocity slip parameter plays an important role in the peristaltic motion of the fluid. It is cleared from figure velocity distribution close the wall are not similar in view of slip parameter. Velocity is increased on increasing slip parameters. Fig. 2(b) shows the impact of $\alpha$ on the bile velocity and it is seen that increasing the value of $\alpha$ has a tendency to boost the velocity as rising $\alpha$ makes fluid less viscous. Fig. 2(c) reveals that in case of no inclination $(\gamma=0)$ bile velocity is minimum and as duct comes under inclined position, velocity increases. Due to the consideration of inclined duct, there would be impact of gravity parameter $\eta$ on the velocity profile so it is obtained from Fig. 2(d) on increasing the magnitude of $\eta$, bile velocity also increases. 

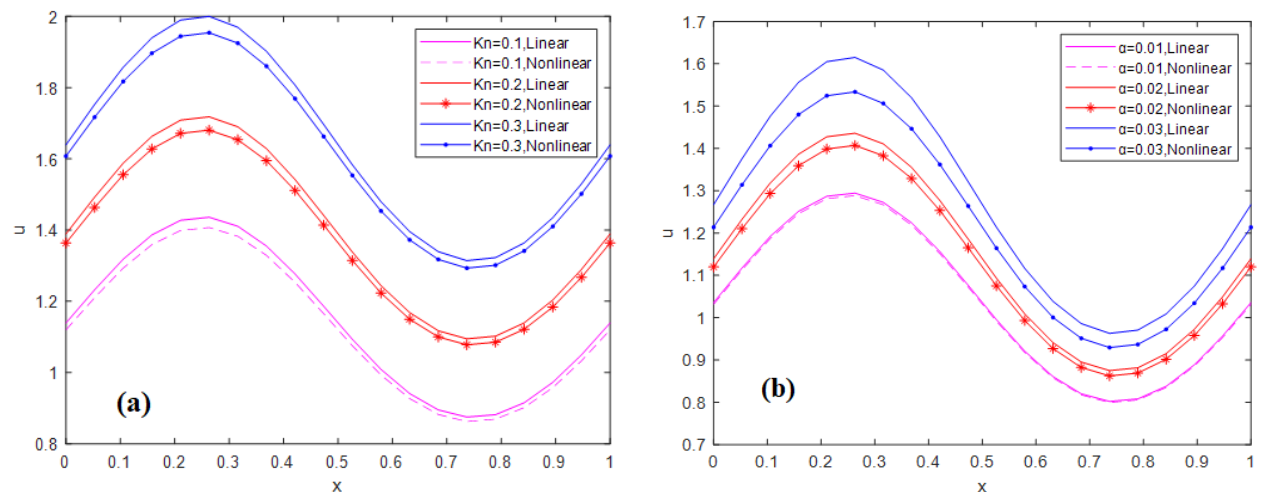

Fig. 2. (a) Plot of velocity $u$ with $x$ for different values of $K_{n}$ with $\alpha=0.1, \gamma=\frac{u}{6}, \eta=1$ and $\phi=0.15$, (b) plot of velocity $u$ with $x$ for different values of $\alpha$ with $K_{n}=0.2, \gamma=\frac{\pi}{6}, \eta=1$ and $\phi=0.15$.
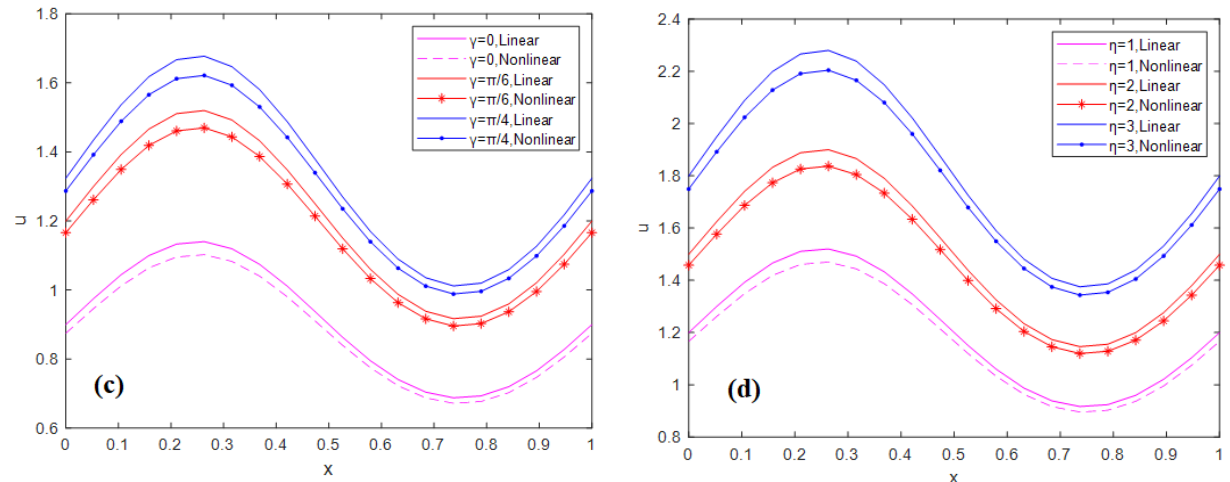

Fig. 2. (c) Plot of velocity $u$ with $x$ for different values of $\gamma$ with $K_{n}=0.2, \alpha=0.1, \eta=$ 1 and $\phi=0.15$ and (d) plot of velocity $u$ with $x$ for different values of $\eta$ with $K_{n}=0.2, \alpha=$ $0.1, \gamma=\frac{\pi}{6}$ and $\phi=0.15$.
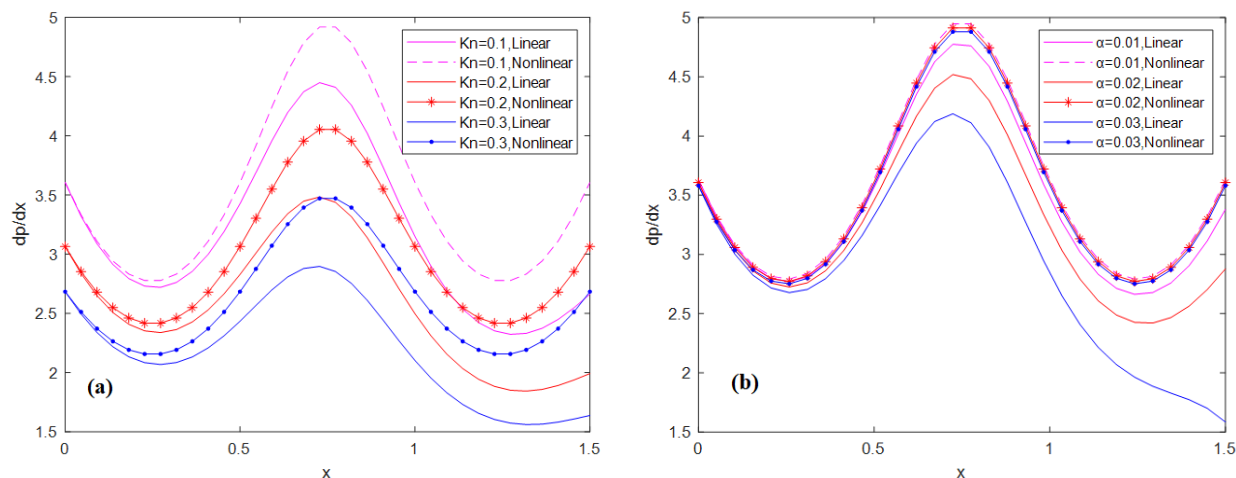

Fig. 3. (a) Plot of pressure gradient $\frac{d p}{d x}$ with $x$ for different values of $K_{n}$ with $\alpha=0.1, \gamma=\frac{\pi}{6}, \eta=$ 1 and $\phi=0.15$ and (b) plot of pressure gradient $\frac{d p}{d x}$ with $x$ for different values of $\alpha$ with $K_{n}=$ $0.2, \gamma=\frac{\pi}{6}, \eta=1$ and $\phi=0.15$. 

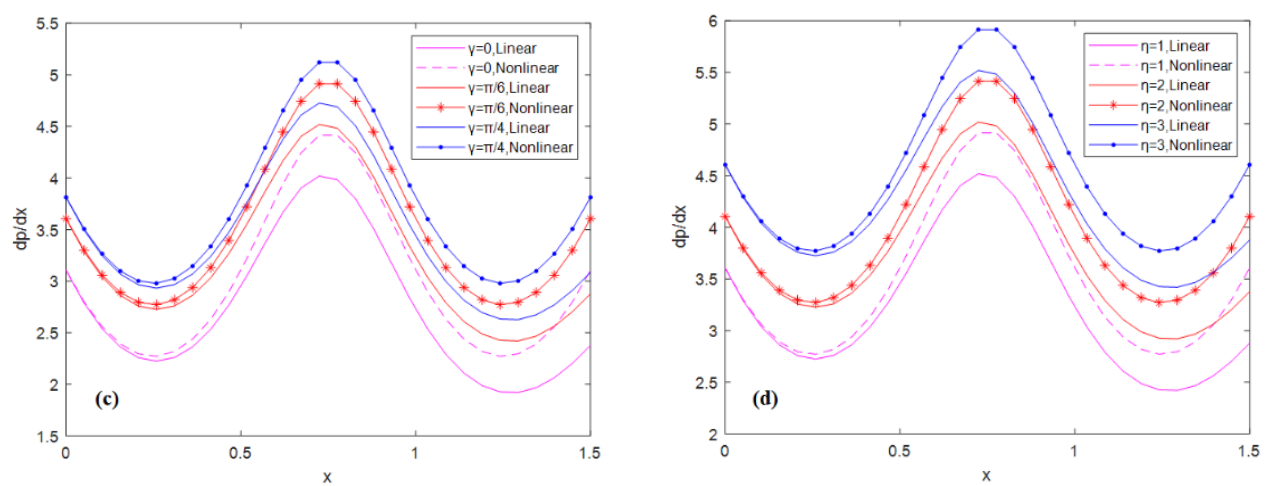

Fig. 3. (c) Plot of pressure gradient $\frac{d p}{d x}$ with $x$ for different values of $\gamma$ with $K_{n}=0.2, \alpha=0.1, \eta=$ 1 and $\phi=0.15$ and (d) plot of pressure gradient $\frac{d p}{d x}$ with $x$ for different values of $\eta$ with $K_{n}=$ $0.2, \alpha=0.1, \gamma=\frac{\pi}{6}$ and $\phi=0.15$.

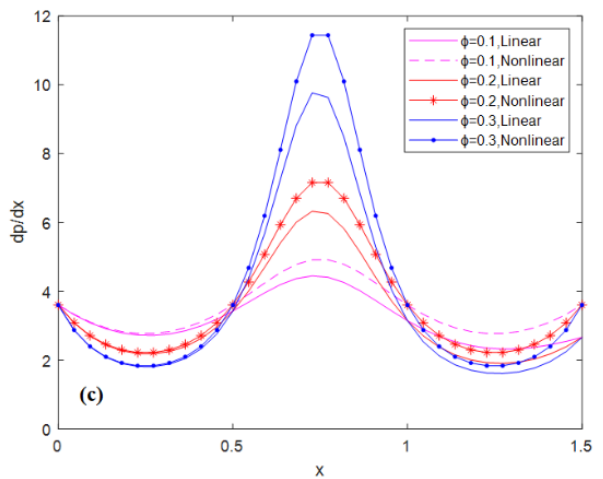

Fig. 3. (e) Plot of pressure gradient $\frac{d p}{d x}$ with $x$ for different values of $\phi$ with $K_{n}=0.2, \alpha=0.1, \gamma=$ $\frac{\pi}{6}$ and $\eta=1$.

The deviation of the pressure gradient $\frac{d p}{d x}$ of bile for different values of angle of inclination is plotted in Fig. 3(c). It is admitted that the pressure gradient increases with increasing $\gamma$ as under inclined position velocity increases; hence more pressure gradient is required to maintain the flow throughout the duct. Fig. 3(d) shows increasing gravity parameter $\eta$ increase pressure gradient. The impact of amplitude ratio $\phi$ is described in Fig. 3(e). Since the peristaltic motion is taking place, it is observed that the magnitude of the pressure gradient is increased on increasing $\phi$.

The pressure rise $\Delta P$ is calculated in terms of time-average flux $\Theta$, the correlation between pressure rises $\Delta P$ and time-average flux $\Theta$ in a wave frame is displayed from Fig. 4(a-e) for different parameters of the problem angle of inclination $\gamma$, viscosity parameter $\alpha$, gravity parameter $\eta$, velocity slip parameter $K_{n}$ and amplitude ratio $\phi$. All plotted graphs represent the linear relationship between pressure rise $\Delta P$ and time-average flux $\Theta$. It is also observed that pressure rise is more in the case of linear variation of viscosity. The pumping phenomenon can be classified into three regions, where the deviation in pressure rise is carried 
out. The region where $\Delta P>0$ is known as the pumping region and is also known as positive pumping for $\Theta>0$. The region where $\Delta P<0$ is known as the co-pumping region, and $\Delta P=0$ is known as the pumping free region. Fig. 4(a) presents the relationship between pressure rise $\Delta P$ and given flux $\Theta$ for different values of $K_{n}$, it is noticed from here on varying $K_{n}$ in ascending order, required pressure rise $\Delta P$ also increases for the pump to work. Fig. 4(b) reveals the nature of pressure rise $\Delta P$ with time-average flux $\Theta$ for different values of viscosity parameter $\alpha$, it shows pressure rise $\Delta P$ increases with increasing $\alpha$. It is observed from Fig. 4(c) for larger the inclination, the higher-pressure rise $\Delta P$ is required for pumping for given flux $\Theta$. The variation in pressure rises with average flux for $\eta$ is plotted in Fig. 4(d), it is found for larger the gravity parameter $\eta$, the higher-pressure rise works for pumping, it can be seen the increment in pressure rise $\Delta P$ for given flux $\Theta$ with increasing $\eta$, also for given $\Delta P, \Theta$ increases on increasing $\eta$. Fig. 4(e) deals with pressure rise $\Delta P$ for given flux $\Theta$ for $\phi$, it is noticed from here on increasing $\phi$ required pressure rise $\Delta P$ also rises for the pump to work.
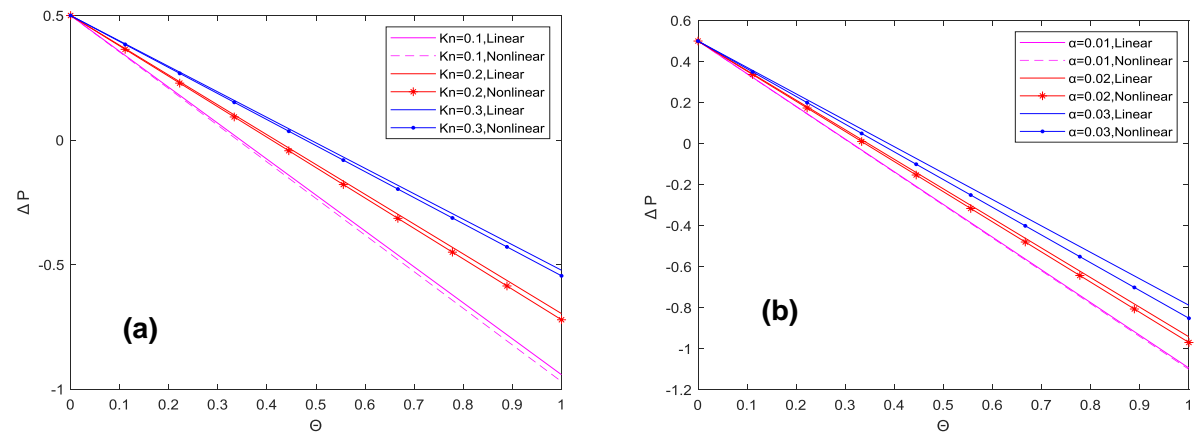

Fig. 4. (a) Plot of pressure rise $\Delta P$ with $\Theta$ for different values of $K_{n}$ with $\alpha=0.1, \gamma=\frac{\pi}{6}, \eta=$ 1 and $\phi=0.15$ and (b) plot of pressure rising $\Delta P$ with $\Theta$ for different values of $\alpha$ with $K_{n}=$ $0.2, \gamma=\frac{\pi}{6}, \eta=1$ and $\phi=0.15$.
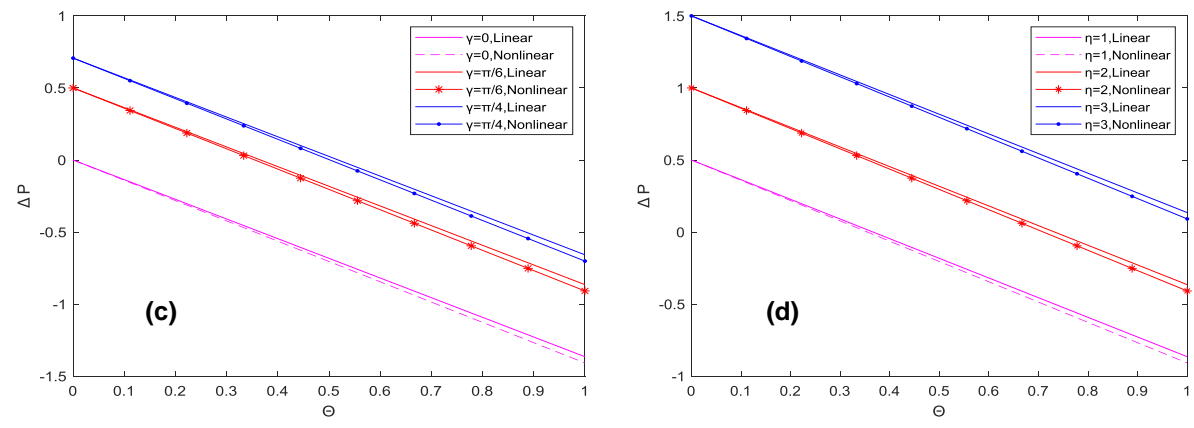

Fig. 4. (c) Plot of pressure rise $\Delta P$ with $\Theta$ for different values of $\gamma$ with $K_{n}=0.2, \alpha=0.1, \eta=$ 1 and $\phi=0.15$ and (d) plot of pressure rise $\Delta P$ with $\Theta$ for different values of $\eta$ with $K_{n}=0.2, \alpha=$ $0.1, \gamma=\frac{\pi}{6}$ and $\phi=0.15$. 


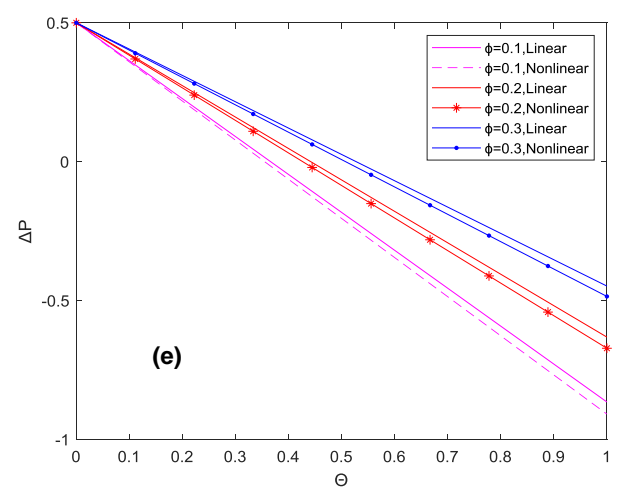

Fig. 4. (e) Plot of pressure rise $\Delta P$ with $\Theta$ for different values of $\phi$ with $\gamma=\frac{\pi}{6}, K_{n}=0.2, \alpha=$ 0.1 and $\eta=1$.

\section{Conclusion}

The present article is designed to analyze the influence of peristaltic transport on bile flow in an inclined duct with variable viscosity and slip boundary conditions. Governing equations of motion have been simplified using long wavelength and low Reynolds number approximation. Closed-form analytic expressions are constructed for axial velocity, pressure gradient and pressure rise. The influence of various parameters on axial velocity, pressure gradient and pressure rise are drawn with MATLAB R2018b software. This article has a physical significance because, in a diseased state, bile thickness increases and bile becomes more viscous, leading to a change in bile viscosity. The following points are made to summarize the study

- It is clear from the graphs in the case of nonlinear variation of viscosity, less velocity is noted down.

- Bile velocity increases on increasing inclination parameter, viscosity parameter, gravity parameter and velocity slip parameter.

- Graphs show that in the case of nonlinear variation of viscosity, more pressure gradient is essential to flow the bile throughout the duct.

- It is possible to boost pressure rise $\Delta P$ for all effecting parameters of the model.

\section{References}

1. T. W. Latham, Master's Thesis, MIT, Cambridge, 1996.

2. T. Hayat and N. Ali, Appl. Math. Model. 32, 761 (2008). https://doi.org/10.1016/j.apm.2007.02.010

3. P. Muthu, B. V. R. Kumar, and P. Chandra, Appl. Math. Model. 32, 2019 (2008). https://doi.org/10.1016/j.apm.2007.06.034

4. Y. A. Elmaboud, K. Mekheimer, and S. I. Abdelsalam, Appl. Bionics Biomech. 11, 197 (2014). https://doi.org/10.1155/2014/618637

5. N. S. Akbar, T. Hayat, S. Nadeem, and S. Obaidat, Int. J. Heat Mass Transfer 55, 1855 (2012). https://doi.org/10.1016/j.ijheatmasstransfer.2011.11.038 


\section{Modeling of Nonlinear Variable Viscosity of Fluid}

6. A. Kavitha, R. H. Reddy, R. Saravana, and S. Sreenadh. Ain Shams Eng. J. 8, 683 (2017). https://doi.org/10.1016/j.asej.2015.10.014

7. A. Tanveer, T. Hayat, and A. Alsaedi, Appl. Math. Mech. 39, 501 (2018). https://doi.org/10.1007/s10483-018-2313-8

8. A. A. Khan, R. Ellahi, and M. Usman, J. Porous Media. 16, 59 (2013). https://doi.org/10.1615/JPorMedia.v16.i1.60

9. M. Gottschalk and A. Lochner, Gastoenterologisches J. 50, 65 (1990).

10. M. Al-Atabi, S. B. Chin, and X. Y. Luo, J. Biomech. Eng. 132, ID 041003 (2012). https://doi.org/10.1115/1.4001043

11. M. Al-Atabi, R. C. Ooi, X. Y. Luo, S. B. Chin, and N. C. Bird, Med. Eng. Phys. 34, 1177 (2012). https://doi.org/10.1016/j.medengphy.2011.12.006

12. A. G. Kuchumov, Y. I. Nyashin. and V. A. Samartsev, 7th WACBE World Congr. Bioeng. 52, 158 (2013). https://doi.org/10.1007/978-3-319-19452-3 42

13. X. Luo, W. Li, N. Bird, S. B. Chin, N. A. Hill, and A. G. Johnson, World J. Gastroenterol. 13, 1384 (2007). https://doi.org/10.3748/wjg.v13.i9.1384

14. R. C. Ooi, X. Y. Luo, S. B. Chin, A. G. Johnson, and N. C. Bird, J. Biomech. 37, 1913 (2004). https://doi.org/10.1016/j.jbiomech.2004.02.029

15. A. G. Kuchumov, V. Gilev, V. Popov, V. Samartsev, and V. Gavlilov, Korea Aust. Rheol. J. 26, 81 (2014). https://doi.org/10.1007/s13367-014-0009-1

16. A. G. Kuchumov, Y. I. Nyashin, V. A. Samarcev, and V. A. Gavrilov, Acta Bioeng. Biomec. 15, 9 (2013).

17. A. G. Kuchumov, Russ J. Biomech. 20, 77 (2016).

18. S. Maiti and J. C. Misra, Int. J. Eng. Sci. 49, 950 (2011). https://doi.org/10.1016/j.ijengsci.2011.05.006

19. K. Gujral and S. P. Singh, Int. J. Comput. Sci. Math. 11, 397 (2020). https://doi.org/10.1504/IJCSM.2020.107603

20. J. S. Goud, P. Srilatha, L. Indira, B. Raju, and A. Praveen, J. Nat. Remedies 21, 18 (2020).

21. W. Mahmood, M. Sajid, M. N. Sadiq, and N. Ali, Pramana 95, ID 7 (2021). https://doi.org/10.1007/s12043-020-02055-Z

22. A. Sinha, G. C. Shit, and N. K. Ranjit, Alex. Eng. J. 54, 691 (2015). https://doi.org/10.1016/j.aej.2015.03.030 